\title{
CALIDAD DEL ARQUEO DE CAJA MEDIANTE EL USO DE UNA APP MOVIL
}

\section{QUALITY OF CASH TACKLE THROUGH THE USE OF A MOBILE APP}

\author{
Dr. Juan Pedro Benítez Guadarrama ${ }^{a}$ \\ Dra. Ana Luisa Ramírez Roja ${ }^{\text {b }}$ \\ Mtro. Jorge Enrique Zamora Pérez ${ }^{c}$
}

\begin{abstract}
${ }^{a}$ Universidad Autónoma del Estado de México Facultad de Contaduría,jpbenitezg@uaemex.mx

${ }^{\mathrm{b}}$ Universidad Autónoma del Estado de México Facultad de Contaduría, alramirezr@uaemex.mx ${ }^{\mathrm{c}}$ Corporativo Universitario México Posgrado,jezp13@prodigy.net.mx
\end{abstract}

\section{RESUMEN}

Ante el incremento acelerado de aplicaciones móviles en plataformas digitales se observan herramientas tecnológicas que pretenden ser útiles al usuario en la solución inmediata en situaciones laborables, académicas o sociales; al momento de descargar o adquirir las aplicaciones móviles para realizar una tarea o una actividad determinada, se piensa en obtener los resultados sobre la información que se requiere o se solicita; el objetivo de esta investigación es mostrar el nivel de calidad que tiene la App como herramienta para generar información financiera sobre el manejo y control del efectivo; en el desarrollo de la aplicación se sustentó en la norma ISO 13407, esta norma establece el diseño centrado en las necesidades del usuario y los procedimientos para el procesamiento de datos con base en la normatividad de auditoría en donde se sustenta el control interno del rubro sobre el efectivo; para realizar las pruebas sobre el uso de la aplicación se utilizaron datos reales de pymes dedicados a la actividad comercial, industrial y prestación de servicios; en la validación de contendió y resultados participaron 80 contadores públicos certificados, quienes dieron su opinión por escrito sobre el nivel de uso conforme a la norma ISO 25010; los resultados revelan que la herramienta presenta niveles de calidad excelentes debido a que cumple con todas y cada una de las variables de funcionalidad, eficiencia, compatibilidad, usabilidad, confiabilidad, seguridad, mantenibilidad y portabilidad, por 
tanto es generador de información financiera oportuna, confiable, comprensible y relevante, útil al usuario para una efectiva toma de decisiones en el manejo y control del efectivo.

PALABRAS CLAVE: Calidad, Efectivo, App móvil.

\begin{abstract}
In view of the accelerated increase of mobile applications in digital platforms, technological tools are observed that are intended to be useful to the user in the immediate solution in working, academic or social situations; at the time of downloading or acquiring the mobile applications to perform a task or a certain activity, we think about obtaining the results on the information that is required or requested; The objective of this research is to show the level of quality that the App has as a tool to generate financial information about the management and control of cash; in the development of the application was based on ISO 13407, this standard establishes the design focused on the needs of the user and the procedures for data processing based on the audit regulations where the internal control of the item on the cash is supported; In order to carry out the tests on the use of the application, real data from SMEs dedicated to commercial, industrial and service provision activities were used; 80 certified public accountants participated in the validation of the contents and results, and gave their opinion in writing on the level of use according to the ISO 25010 standard; The results reveal that the tool presents excellent quality levels due to the fact that it fulfills each and every one of the functionality, efficiency, compatibility, usability, reliability, security, maintainability and portability variables, therefore it is a generator of timely, reliable, understandable and relevant financial information, useful to the user for effective decision making in the management and control of cash.
\end{abstract}

KEYWORDS: Quality, Cash, mobile app.

\title{
I. INTRODUCCIÓN
}

Ante el incremento acelerado de aplicaciones móviles que se adicionan en las plataformas digitales con el propósito de ser herramientas útiles al usuario para la solución inmediata de situaciones laborables, académicas o sociales; el usuario realiza la búsqueda de una aplicación con la intención de descargar o adquirir la App para realizar una tarea o una 
actividad encomendada, se espera que el usuario obtenga aplicaciones móviles con contenidos exigibles por las normas de información financiera que aseguren el procesamiento de datos y una estructura integral que genere y presente información confiable, relevante, comprensible y sea útil al usuario para mejorar el control sobre el recurso financiero, por tal motivo el propósito de esta la investigación es mostrar el nivel de calidad que tiene la App en la práctica como un recurso generador de información financiera sobre el manejo y control del efectivo; para el desarrollo de la aplicación se empleó el procedimiento establecido en la norma internacional de la calidad ISO 13407, en el documento se establece que cualquier procedimiento que realice por el diseñador para el desarrollo de la aplicación móvil debe apegarse a las necesidades y requerimientos del usuario; en la realización de las pruebas se tomaron datos reales de pequeñas y medianas empresas de diferentes sectores empresariales a fin de utilizar la herramienta tecnológica con datos reales; para la evaluación de la aplicación fue utilizada por la participación activa de contadores públicos certificados quienes son especialistas y conocedores de la normatividad sobre el manejo y control del efectivo, dieron su opinión por medio de la aplicación de un instrumento, conformado por variables contendidas por la norma ISO 25010; los resultados muestran que la herramienta presenta niveles de calidad excelentes debido a que cumple con todas y cada una de las variables de funcionalidad, eficiencia, compatibilidad, usabilidad, confiabilidad, seguridad, mantenibilidad y portabilidad, por tanto se puede inferir que la información generada y presentada en la aplicación móvil cumple con las disposiciones contendidas en la normatividad financiera y los requerimientos y necesidades del usuario para informar sobre el manejo y control del efectivo.

Piattini et al., (2006) afirma que la calidad es un elemento indispensable aplicado a las características que se manifiestan como atributos de los software en cualquier aplicación tecnológica, mientras que la Real Academia Española (2020), define a la calidad, "como la propiedad o conjunto de propiedades inherentes a algo, que permiten juzgar su valor', (s.f., definición1); la International Standards Organization, ISO en la norma 8402:1994, la define como los atributos que debe poseer un producto o servicio que garanticen la satisfacción de los usuarios al cubrir las necesidades solicitadas ya sea que hayan sido expresadas o no; en la actualización de la Norma ISO, la 9000:2005, la define como el cumplimiento total o 
parcial de atributos que se especifican. Pressman (2010) la considera como las características que se especifican referentes a las necesidades de funcionalidad y rendimiento, que cumplan con esquemas establecidos de satisfacción en los usuarios que se buscan para software desarrollados profesionalmente; Monsalve, (2014) especifica que los productos que poseen calidad detallan caracteres específicos positivos, que dan el soporte de la calidad y no precisamente se detalla como una cualidad. Las aplicaciones móviles o apps han tenido gran aceptación en las diferentes actividades sociales, en las actividades laborales es común que los empresarios se enfrenten a cuestiones como ¿Cuánto tengo de efectivo? ¿Cómo se maneja el efectivo?, ¿Cuánto es el importe de monedas, billetes o documentos?, ¿Cuánto es el saldo al final de periodo?, ¿Existe alguna diferencia entre el registro en libros con el saldo final del efectivo?; interrogantes que solo se responden cuando existe un control del recurso financiero efectivo, con la finalidad de tener una respuesta de manera inmediata y cuando se requiera la información por el usuario en las actividades empresariales, es común que los empresarios o representantes legales contraten a una persona externa para verificar el cumplimiento de los controles internos en la organizaciones o negocio, un especialista en el contexto financiero, es el encargado de llevar acabo las verificaciones y procedimientos para el cumplimiento sobre el manejo y control del recurso financiero en las organizaciones, ante este fenómeno se ha desarrollado una aplicación financiera denominada arqueo de caja, para ser utilizada como herramienta de apoyo a fin de comprobar el saldo inicial y final del efectivo, así como la existencia del efectivo (monedas y billetes ) y los comprobantes fiscales, el propósito es evidenciar el manejo, responsabilidad y aplicación correcta de las políticas monetarias de la organización y brindar información, inmediata, precisa, oportuna y exacta, sustentada con disposiciones contenidas en las guías de auditoria en el boletín 6100 y las Normas de Información Financiera, NIF C-1 "Efectivo y equivalente de efectivo" para el ejercicio 2020.

Los objetivos son los siguientes:

- Describir el procedimiento de la investigación para conocer el nivel de calidad que tiene la App en casos financieros sobre el arqueo de caja.

- Medir el nivel de las variables independientes de funcionalidad, compatibilidad, 
eficiencia, confiabilidad, mantenibilidad, portabilidad y usabilidad en relación con la variable de calidad del software.

- Describir los resultados sobre la aplicación del instrumento de evaluación con el propósito de cumplir con las variables que conforman a la calidad.

\section{MARCO REFERENCIAL}

ABC Economía (2013) señala que Javier Rodríguez director general de Google España refiere que en una encuesta realizada por la telefonía Jobandtalent a más de 2,500 usuarios, el $72 \%$ de los usuarios afirma que utiliza aplicaciones móviles como herramienta útil para sus trabajos, facilitan la organización, la consulta rápida, la interactuación instantánea; en el informe revela que usan aplicaciones para su trabajo, en cinco áreas profesionales: banca y Finanzas, Profesionales de la Comunicación, Diseñadores gráficos, Profesionales de la investigación científica, y profesionales de la Medicina y Farmacia, 12\% afirma haber descargado alguna aplicación relacionada con su trabajo, pero no la utiliza, el 16\% declara utilizar ninguna App que favorezca o se complemente con el desarrollo laboral, el importante nicho laboral que hay que aprovechar.

IAB México (2012) con apoyo de Mobext y Terra realizo el primer estudio en México con la finalidad de conocer los usos y hábitos de las personas que poseen dispositivos móviles, con base en cifras de la COFETEL (Comisión Federal de Telecomunicaciones), destaca que de un total de 95.5 millones de líneas telefónicas celulares, el $85 \%$ representa a los mexicanos que poseen un dispositivo móvil, de los que el $17 \%$ pertenece a smartphone y destaca que el $83 \%$ es celular convencional, con respecto a las Tabletas informa que su uso está por debajo del 5\%; el 91\% de los usuarios utiliza el equipo para llamadas telefónicas, el 70\% de ellos lo utiliza para tener entretenimiento; un grupo más limitado es el de usuarios que navegan en internet con un $38 \%$, y tan solo el $21 \%$ utiliza estos dispositivos para actividades especializadas( noticias, abrir archivos del trabajo, y realizar transacciones bancarias). 
Algunos estudios realizados por Benítez \& Ramírez (2013) sobre la evaluación de calidad de la aplicaciones móvil para determinar la PTU en 120 empresas mexicanas del estado de México, a través de la metodología norma ISO 9126, obtuvieron excelente niveles de calidad en la aplicación, concluyen que "puede ser una herramienta tecnológica móvil que consigue incorporase en la gestión empresarial y su relación con las autoridades fiscales", hasta el momento no se ha encontrado literatura acerca de la evaluación de este tipo de tecnologías en el campo laboral.

Todo modelo o estándar de calidad en un ámbito laboral tiene una aplicación específica y posee como finalidad el mejoramiento continuo en las actividades laborales, una vez usada e implantada puede ser medible a través de modelos y estándares de calidad del software, los cuales deben reunir atributos que ayuden a realizar actividades y funciones de forma que puedan planearse, controlarse y ejecutarse de un modo formal y sistemático (Scalone, 2006). Solarte, Muñoz \& Arias (2009) sostienen que las cualidades de una aplicación deben ser medibles y específica, dependiendo del tipo de estudio en el que se trabaje, teniendo como finalidad la utilidad y su existencia, buscando que se pueda mantener los diferentes costos que se desarrollan conforme las etapas de la aplicación.

Algunos estudios muestran que la tecnología proporciona seguridad, simplificación, precisión, y confianza en las actividades profesionales en la organización; Moreno, González \& Echartea (2008) evaluó la calidad de espacios virtuales con el modelo ISO/IEC 9126-4 con la denominación de SW-AQUA, este modelo refiere a 4 aspectos en el Instituto tecnológico de Motul, el primero refiere a la efectividad, el segundo a la productividad, el tercero a la seguridad y el ultimo a la satisfacción la muestra se llevo acabo con la participación de 8 sujetos dicho estudio demostró ser un sitio funcional, rápido, reducción de errores, exacto, preciso y confiable para el usuario. Omaña y Cárdenas (2010) realizó una estudio documental no experimental, descriptivo y transaccional donde evaluó un software denominado SQLfi, mediante el modelo sistémico de calidad del software (MOSCA) este estudio se aplicó abordando a 26 sujetos con una muestra intencional de 11, Su resultado fue un nivel sistémico de calidad nulo, por lo que propone la adopción de un modelo de desarrollo para la 
construcción de software de calidad basado en estándares establecidos, por su parte Santoveña (2010) diseñó un instrumento de la calidad de los cursos virtuales de la UNED en España, el instrumento consta de 36 items organizado en tres dimensiones: Calidad general del entorno y metodología, calidad técnica (navegación y diseño) y la calidad de recursos multimedia; a fin de presentar una propuesta de mejora. Rodríguez et al., (2010) presenta una metodología para la evaluación de la calidad en los modelos UML, formada por un conjunto estructurado de procesos orientado a la evaluación de la calidad, donde a partir de una revisión de estándares, normas y metodologías relacionadas con la evaluación de la calidad del software, elaboró la metodología EVVE, bajo los siguientes principios: formada por un conjunto estructurado de procesos, orientado a la relación con el cliente y la externalización de la evaluación de la calidad, fácilmente adaptable; sin embargo identifica el qué, cuándo, y el quién, de cada una de las fases y actividades de los procesos, así como la secuencia de pasos que se debe seguir a la hora de llevar a cabo la evaluación.

Solarte, Muñoz \& Arias (2009) realizó un análisis de los modelos de calidad entre la norma ISO/IEC, la integración del modelo de maduración de la capacidad CMMI y el modelo de calidad IT MARK para las PYMES, para determinar los beneficios e inconvenientes que presenta el desarrollo de software con calidad; infiere que el modelo CMMI ayuda a encontrar la mejor manera de trabajar, no detalla los procesos, tiene que reforzarse y usarse apropiadamente; El modelo ISO/IEC 15504 identifica los riesgos de los costos, mejora la eficiencia y calidad además de evaluar los procesos de software; IT MARK es el primer modelo de calidad internacional diseñado para las PYMES en base a un conjunto de herramientas desvinculadas entre sí, generando dificultad para gestionarlas, mantenerlas y controlarlas, con base en estos modelos se podrá adoptar los requerimientos exigibles por la actividad profesional.

El presente trabajo se sustenta en el modelo de calidad establecido por el estándar ISO 25010 publicado en 1992 bajo el nombre de Information technology-Software product evaluation: Qualitycharacteristics and guide linesfortheir use, dicho modelo sustenta a la calidad en términos de la funcionalidad, confiabilidad, usabilidad, eficiencia, mantenibilidad y portabilidad. 


\section{METODOLOGÍA, TÉCNICAS Y MATERIALES EMPLEADOS}

El diseñó de la App financiera contiene disposiciones normativas establecidas en las guías de auditoría sobre el control interno del efectivo y reglas de valuación y revelación establecidas en las NIF C1 para el ejercicio 2020, esta aplicación tecnológica móvil puede ser utilizada en teléfonos inteligentes, tabletas electrónicas y en computadoras con emuladores con sistema operativo Android, la aplicación proporciona información sobre el arqueo de caja, considerado como el recuento físico del efectivo y comprobantes que el encargado de caja maneja y controla, con esta información el usuario da cumplimiento a la normatividad y además justifica la aplicación del control interno; para su evaluación se creó un instrumento de medición de la calidad basado en los criterios e indicadores en la Norma ISO 25010 conformado por 31 ítems (tabla 1) estructurado a escala tipo Likert, considerando 1 como excelente, 2 bueno, 3 aceptable, deficiente, 5 malo.

Tabla 1.

Variables e indicadores de la calidad.

\begin{tabular}{|c|c|c|}
\hline Ítem & INDICADOR & VARIABLE \\
\hline 1 & Completitud & \multirow{3}{*}{ Funcionalidad } \\
\hline 2 & Corrección & \\
\hline 3 & Pertinencia & \\
\hline 4 & Comportamiento & \multirow{3}{*}{ Eficiencia } \\
\hline 5 & Utilización & \\
\hline 6 & Capacidad & \\
\hline 7 & Coexistencia & \multirow[t]{2}{*}{ Compatibilidad } \\
\hline 8 & Inter operatividad & \\
\hline 9 & Inteligibilidad & \multirow{6}{*}{ Usabilidad } \\
\hline 10 & Aprendizaje & \\
\hline 11 & Operatividad & \\
\hline 12 & Protección & \\
\hline 13 & Estética & \\
\hline 14 & Accesibilidad & \\
\hline 15 & Madurez & \multirow{4}{*}{ Fiabilidad } \\
\hline 16 & Disponibilidad & \\
\hline 17 & Tolerancia a fallos & \\
\hline 18 & Recuperación & \\
\hline 19 & Confiabilidad & \multirow[b]{3}{*}{ Seguridad } \\
\hline 20 & Integridad & \\
\hline 21 & No repudio & \\
\hline
\end{tabular}




\begin{tabular}{|c|c|c|}
\hline 22 & Autenticidad & \\
\hline 23 & Responsabilidad & \\
\hline 24 & Modularidad & \\
\hline 25 & Reusabilidad & \multirow{2}{*}{ Mantenibilidad } \\
\hline 26 & Analizabilidad & \\
\hline 27 & Modificación & \\
\hline 28 & Prueba & \\
\hline 29 & Adaptabilidad & Portabilidad \\
\hline 30 & Instalación & \\
\hline 31 & Remplazo & \\
\hline
\end{tabular}

Fuente: Elaboración propia con base en la NOM ISO 25010 (2019).

Una vez diseñado el instrumento para medir la calidad de la App, se realizó el proceso de la evaluación en cinco etapas, con base en los procedimientos establecidos por la norma ISO 14958 en el apartado 5, el instrumento se aplicó a 80 participantes todos fueron contadores públicos certificados que desempeñan actividades en el área financiera, se encuentran de manera activa al interior de las organizaciones en el municipio de Ecatepec de Morelos del Estado de México.

Al inicio del procedimiento se indicó a los participantes que la finalidad de la práctica es evaluar la App financiera, posteriormente se explicó que es una aplicación que deben instalar en sus dispositivos móviles (teléfono inteligente, tabletas, PC) con sistema operativo Android, se puntualizó sobre el propósito principal de la herramienta el cual es brindar información sobre el recuento físico del efectivo y compararla con el registro en libros de contabilidad, para que los empresarios puedan dar cumplimiento preciso y oportuno a la normatividad financiera vigente. Así mismo, se especificó que el desarrollo del modelo tecnológico fue diseñado y elaborado con base en las guías de auditoria y la NIF C1. Posteriormente se proporcionó la app por medio de bluetooth a los teléfonos inteligentes y tabletas personales para la revisión del caso práctico y finalmente concluir con el proceso de evaluación.

Para la operacionalización metodológica se describe cada una de las fases o etapas en las que se llevó a cabo el método para fundamentar y justificar el cumplimiento del objetivo de la investigación y dar a conocer los resultados de los hallazgos de los recursos tecnológicos puestos en práctica en casos específicos que afectan o que impone la normatividad 
financiera mexicana:

\section{Primera Fase}

En esta etapa se indicó a los participantes la finalidad de la práctica, la cual era evaluar la App financiera, diseñada para presentar información sobre el recuento físico del efectivo y sus equivalentes de efectivo, así como los comprobantes de las erogaciones realizadas en un periodo determinado.

\section{Segunda Fase}

En esta fase se proporcionó la herramienta a los participantes por medio de bluetooth, asiendo de su conocimiento que no es el único medio por el cual se puede acceder a ella que también se puede compartir por medio de otros canales como WhatsApp, correo electrónico o descargándola de la play store; se solicitó que una vez obtenida, se iniciara el proceso de instalación en sus dispositivos móviles, para continuar con su utilización en el caso práctico.

\section{Tercera Fase}

En esta fase se les explico cada uno de los elementos y conceptos que estructuran la aplicación destacando que para los participantes todos los elementos eran de su comprensión y se encontraban asociados con los rubros que conforman cada uno de los apartados, los participantes son expertos en el control interno y el registro de las operaciones.

\section{Cuarta Fase}

En esta fase se les solicito a los participantes realizar el caso práctico mediante la utilización de la App sobre el arqueo de caja, con el fin |de utilizar, verificar y comprobar que los resultados presentados por la herramienta tecnológica son confiables, reales, verídicos y comprobables para ser comparables con los saldos de los libros de contabilidad.

\section{Quinta Fase}


En esta fase se destaca que una vez que se utilizó y aplico la app por los participantes, se les proporciono el instrumento de evaluación con la finalidad de conocer su opinión y precepción de los contadores públicos sobre la aplicación para generar, presentar, guardar y enviar información.

Al realizar el procesamiento y análisis de los datos, se utilizó el paquete estadístico Statical Package for the Social Sciencies (SPSS, Versión $20.0^{\circledR}$ ), programa estadístico informático usado como instrumento de análisis cuantitativo que facilita el manejo de los datos obtenidos en la investigación de campo, mediante la utilización del programa se realizó lo siguiente:

Con la aplicación del análisis descriptivo se tendrá el análisis de las medidas de tendencia central en los datos sociodemográficos por género, edad, marca de celular, actividad y finalmente por cada uno de los criterios que miden la calidad.

Con la aplicación del análisis inferencial, se argumentara sobre las afirmaciones de los participantes mediante la correlación de Pearson (r) con el objetivo de encontrar el grado de relación existente entre dos variables, la regresión lineal simple (Y) predice la influencia que tiene una variable con respecto a la otra y el coeficiente de determinación $\left(\mathrm{r}^{2}\right)$, con el propósito de conocer la proporción de la variación entre la variable independiente con respecto a la variable dependiente, la cual sirvió para medir los niveles de calidad de la aplicación móvil en la práctica financiera.

\section{RESULTADOS Y DISCUSIÓN}

La muestra fue elegida de una población de 80 sujetos, donde el 100\% ( $\mathrm{n}=80)$ aceptaron voluntariamente participar en el estudio. El 60\% $(n=48)$ eran hombres y el 40\% (n=32) mujeres quienes emplearon la aplicación financiera en su teléfono inteligente como herramienta para verificar el control sobre el manejo del efectivo en las organizaciones (tabla 2). 


\section{Tabla 2.}

Distribución de la muestra por género.

\begin{tabular}{ccc}
\hline Genero & Frecuencia & Porcentaje \\
\hline Femenino & 32 & 40 \\
\hline Masculino & 48 & 60 \\
\hline Total & 80 & 100.0
\end{tabular}

Fuente: Elaboración propia (2019).

Por lo que respecta a la edad de los participantes, el $20.0 \%(\mathrm{n}=16)$ tenían 39 años, el $20.0 \%(\mathrm{n}=16)$ tenían 40 años, el $20.0 \%(\mathrm{n}=16)$ tenían 41 años, el 20.0\% ( $\mathrm{n}=16)$ tenían 42 años y el $20.0 \%(n=16)$ tenían 43 años (Tabla 3).

\begin{tabular}{lcc} 
Tabla 3. \\
Distribución de la muestra por edad. \\
\hline Edad & Frecuencia & Porcentaje \\
\hline 39 años & 16 & $20.0 \%$ \\
\hline $\mathbf{4 0}$ años & 16 & $20.0 \%$ \\
\hline $\mathbf{4 1}$ años & 16 & $20.0 \%$ \\
\hline $\mathbf{4 2}$ años & 16 & $20.0 \%$ \\
\hline $\mathbf{4 3}$ años & 16 & $20.0 \%$ \\
\hline Total & 80 & $100.0 \%$ \\
\hline
\end{tabular}

Fuente: Elaboración propia (2019).

Del total de la muestra, los participantes usaron celulares distintos: el 30\% $(\mathrm{n}=24)$ usaron Huawei, el 30\% ( $n=24)$ LG, el 30\% ( $n=24)$ Samsung, y el 10\% ( $n=8)$ Nokia (Tabla 4).

\section{Tabla 4.}

Distribución por marca de celular

\begin{tabular}{ccc}
\hline Marca de celular & Frecuencia & Porcentaje \\
\hline Huawei & 24 & $30 \%$ \\
\hline LG & 24 & $30 \%$ \\
\hline Samsung & 24 & $30 \%$ \\
\hline Nokia & 8 & $10 \%$ \\
\hline Total & 80 & $100 \%$
\end{tabular}


Fuente: Elaboración propia (2019).

Respecto al tipo de servicios que prestan los participantes, el $30 \%(n=24)$ se dedican a prestan servicios al comercio, el $30.0 \%(\mathrm{n}=24)$ prestan servicio al sector servicios y el 40 $\%(\mathrm{n}=32)$ prestan servicios al sector de transformación (Tabla 5).

Tabla 5.

Distribución por prestación de servicios.

\begin{tabular}{ccc}
\hline Servicios & Frecuencia & Porcentaje \\
\hline Comercio & 24 & $30.0 \%$ \\
\hline Servicios & 24 & $30.0 \%$ \\
\hline Transformación & 32 & $40.0 \%$ \\
\hline Total & 80 & $100.0 \%$
\end{tabular}

Fuente: Elaboración propia (2019).

Los criterios de evaluación descriptivos: Media ( $\mu$ ), Mediana (Me), Moda (Mo) y Desviación Estándar $(\sigma)$, factores predictores de la calidad, muestran que el nivel de calidad global (puntuación total); en cada una de sus subescalas: Funcionalidad $(\mu=1.20 ; \mathrm{Me}=1.00$; $\left.\mathrm{Mo}=1.00 ; \sigma^{2}=0.162 ; \sigma=0.40\right)$, eficiencia $\left(\mu=1.16 ; \mathrm{Me}=1.00 ; \mathrm{Mo}=1.00 ; \sigma^{2}=0.051 ; \sigma=0.22\right)$, compatibilidad $\left(\mu=1.18 ; \mathrm{Me}=1.00 ; \mathrm{Mo}=1.00 ; \sigma^{2}=0.077 ; \sigma=0.27\right)$, usabilidad $(\mu=1.18$; $\left.\mathrm{Me}=1.00 ; \mathrm{Mo}=1.00 ; \sigma^{2}=0.077 ; \sigma=0.27\right)$, confiabilidad $(\mu=1.17 ; \mathrm{Me}=1.00 ; \mathrm{Mo}=1.00 ;$ $\left.\sigma^{2}=0.061 ; \quad \sigma=0.24\right), \quad$ seguridad $\left(\mu=1.18 ; \quad \mathrm{Me}=1.00 ; \quad \mathrm{Mo}=1.00 ; \sigma^{2}=0.083 ; \quad \sigma=0.28\right)$, mantenibilidad $\left(\mu=1.17 ; \mathrm{Me}=1.00 ; \mathrm{Mo}=1.00 ; \sigma^{2}=0.078 ; \sigma=0.27\right)$ y portabilidad $(\mu=1.17$; $\left.\mathrm{Me}=1.00 ; \mathrm{Mo}=1.00 ; \sigma^{2}=0.082 ; \sigma=0.28\right)$, tienen un nivel excelente de calidad $(\mu=1.17$; $\left.\mathrm{Me}=1.00 ; \mathrm{Mo}=1.00 ; \sigma^{2}=0.071 ; \sigma=0.26\right)$ (Tabla 6).

Tabla 6.

Factores predictores de la calidad.

\begin{tabular}{cccccc}
\hline Factores & $\begin{array}{c}\text { Media } \\
(\boldsymbol{\mu})\end{array}$ & $\begin{array}{c}\text { Mediana } \\
(\mathbf{M e})\end{array}$ & $\begin{array}{c}\text { Moda } \\
(\mathbf{M o})\end{array}$ & $\begin{array}{c}\text { Varianza } \\
\left(\boldsymbol{\sigma}^{2}\right)\end{array}$ & $\begin{array}{c}\text { Desviación } \\
\text { estándar }(\boldsymbol{\sigma})\end{array}$ \\
\hline Funcionalidad & 1.2000 & 1.00 & 1.00 & 0.162 & 0.40252 \\
\hline Eficiencia & 1.1625 & 1.00 & 1.00 & 0.051 & 0.22498 \\
\hline $\begin{array}{c}\text { Compatibilida } \\
\text { d }\end{array}$ & 1.1813 & 1.00 & 1.00 & 0.077 & 0.27837 \\
\hline Usabilidad & 1.1813 & 1.00 & 1.00 & 0.077 & 0.27837 \\
\hline
\end{tabular}




\begin{tabular}{cccccc}
\hline Confiabilidad & 1.1719 & 1.00 & 1.00 & 0.061 & 0.24712 \\
\hline Seguridad & 1.1850 & 1.00 & 1.00 & 0.083 & 0.28777 \\
\hline $\begin{array}{c}\text { Mantenibilida } \\
\text { d }\end{array}$ & 1.1775 & 1.00 & 1.00 & 0.078 & 0.27923 \\
\hline Portabilidad & 1.1750 & 1.00 & 1.00 & 0.082 & 0.28459 \\
\hline CALIDAD & 1.1793 & 1.00 & 1.00 & 0.071 & 0.26584
\end{tabular}

Fuente: Elaboración propia (2019)

Respecto a la correlación de Pearson se observa que existe un grado de relación muy alto entre todas las variables independientes (Funcionalidad $(r=0.81)$, eficiencia $(r=0.90)$, compatibilidad $(r=0.98)$, usabilidad $(r=0.98)$, confiabilidad $(r=0.95)$, seguridad $(r=$ 0.99), mantenibilidad $(\mathrm{r}=0.94)$ y portabilidad $(\mathrm{r}=0.90))$ con respecto a la variable dependiente (calidad), (Tabla 7).

Tabla 7.

Correlación de Pearson.

\begin{tabular}{|c|c|c|c|c|c|c|c|c|c|}
\hline & & \multicolumn{8}{|c|}{ Variables de calidad } \\
\hline & $\begin{array}{c}\text { Funcionalida } \\
\mathrm{d}\end{array}$ & Eficiencia & $\begin{array}{c}\text { Compatibilida } \\
\mathrm{d}\end{array}$ & Usabilidad & $\begin{array}{l}\text { confiabilida } \\
\text { d }\end{array}$ & Seguro & $\begin{array}{c}\text { Mantenibilida } \\
\mathrm{d}\end{array}$ & $\begin{array}{l}\text { Portabilida } \\
\mathrm{d}\end{array}$ & $\begin{array}{c}\text { Calida } \\
\text { d }\end{array}$ \\
\hline Funcionalidad & 1.0 & & & & & & & & \\
\hline Eficiencia & $.662 * *$ & 1.0 & & & & & & & \\
\hline $\begin{array}{c}\text { Compatibilida } \\
d\end{array}$ & $.746^{* *}$ & $\begin{array}{c}.871 * \\
*\end{array}$ & 1.0 & & & & & & \\
\hline Usabilidad & $.746^{* *}$ & $\begin{array}{c}.871 * \\
*\end{array}$ & $1.000^{* *}$ & 1.0 & & & & & \\
\hline Confiabilidad & $.859^{* *}$ & $\begin{array}{c}.952 * \\
*\end{array}$ & $.898 * *$ & $\begin{array}{l}.898 * \\
*\end{array}$ & 1.0 & & & & \\
\hline Seguridad & $.857^{* *}$ & $\begin{array}{c}.859 * \\
*\end{array}$ & $.982 * *$ & $\begin{array}{l}.982 \\
* *\end{array}$ & $.936 * *$ & 1.0 & & & \\
\hline $\begin{array}{c}\text { Mantenibilida } \\
\text { d }\end{array}$ & $.604^{* *}$ & $\begin{array}{c}.852 * \\
*\end{array}$ & $.981 * *$ & $\begin{array}{l}.981 * \\
*\end{array}$ & $.827 * *$ & $928 * *$ & 1.0 & & \\
\hline Portabilidad & $.499^{* *}$ & $\begin{array}{c}.822 * \\
*\end{array}$ & $.950 * *$ & $\begin{array}{l}.950 * \\
*\end{array}$ & $\begin{array}{l}.764 \\
* *\end{array}$ & $\begin{array}{l}.875 \\
* *\end{array}$ & $.992 * *$ & 1.0 & \\
\hline Calidad & $.816^{* *}$ & $.908^{* *}$ & $.989^{* *}$ & $.989 *$ & $.953 * *$ & $\begin{array}{l}.994 * \\
*\end{array}$ & $.947 * *$ & $.902 *$ & 1.0 \\
\hline
\end{tabular}

$* p<0.05 ; * * p<0.01$

Fuente: Elaboración propia (2019).

Respecto a la regresión lineal (Y) se observa que la influencia es significativa y el coeficiente de determinación $\left(\mathrm{r}^{2}\right)$ se observa un proporción de variación significativa entre las variables dependientes (Funcionalidad $\left(\mathrm{Y}=0.81 ; \mathrm{r}^{2}=0.667\right)$, eficiencia $\left(\mathrm{Y}=0.908 ; \mathrm{r}^{2}=\right.$ 0.825), compatibilidad $\left(\mathrm{Y}=0.98 ; \mathrm{r}^{2}=0.978\right)$, usabilidad $\left(\mathrm{Y}=0.98 ; \mathrm{r}^{2}=0.978\right)$, 
confiabilidad $\left(\mathrm{Y}=0.95 ; \mathrm{r}^{2}=0.907\right)$, seguridad $\left(\mathrm{Y}=0.99 ; \mathrm{r}^{2}=0.987\right)$, mantenibilidad $(\mathrm{Y}=$ $\left.0.94 ; \mathrm{r}^{2}=0.89\right)$ y portabilidad $\left.\left(\mathrm{Y}=0.90 ; \mathrm{r}^{2}=0.81\right)\right)$ con respecto a la variable dependiente (calidad), (Tabla 8).

Tabla 8.

Regresión lineal y coeficiente de determinación
Factores
Regresión lineal
Coeficiente de determinación
(Y)
$\left(r^{2}\right)$

\begin{tabular}{cll}
\hline Funcionalidad & 0.816 & 0.667 \\
\hline Eficiencia & 0.908 & 0.825 \\
\hline Compatibilidad & 0.989 & 0.978 \\
\hline Usabilidad & 0.989 & 0.978 \\
\hline Confiabilidad & 0.953 & 0.907 \\
\hline Seguridad & 0.994 & 0.987 \\
\hline Mantenibilidad & 0.947 & 0.898 \\
\hline Portabilidad & 0.902 & 0.813
\end{tabular}

Fuente: Elaboración propia (2019).

La muestra estuvo compuesta por un $40 \%$ de mujeres y $60 \%$ de hombres. En este estudio, aunque existe una variación del $20 \%$ entre los participantes, refleja la misma oportunidad para utilizar la tecnología entre géneros.

Las edades oscilaron entre los 39 a los 43 años, los datos obtenidos representan un equilibrio entre las edades de los participantes del $20 \%$ con una frecuencia de 16 participantes respectivamente entre el rango de edades. Los participantes presentan habilidad en el manejo de la tecnología, por tanto, tienden a la maximización de los recursos tecnológicos, generando confianza y certeza al momento de la evaluación del instrumento.

En el estudio predominó el uso de celular tipo Huawei, LG y Samsung con el $30 \%$ con una frecuencia de 24 objetos, contra el $8 \%$ restante para la marca: Nokia, con una frecuencia de 8. Las características tecnológicas del equipo Huawei, LG y Samsung permiten que estos sean preferencia entre los participantes a diferencia de otras marcas por factores comerciales. 
Así mismo, el 100\% de los participantes están involucrados en la prestación de servicios profesionales en las actividades de comercio con el 30\%, el $30 \%$ a servicios y el $40 \%$ a la transformación de artículos o productos. La variedad en la prestación de servicios profesionales favorece y potencializan la prueba y la evaluación de la aplicación tecnológica debido a la especialización de los contadores en los requerimientos de cada actividad, por tanto, posibilitan la correcta y oportuna presentación de información financiera ante la toma de decisiones en el manejo y control del efectivo.

El instrumento de medición de la calidad de la aplicación móvil obtuvo un Coeficiente Alfa de Cronbach de $\alpha=0.964$, el resultado muestra un nivel de confiabilidad excelente, se infiere que al momento de aplicarlo sin duda mide la calidad de la aplicación móvil puesta en la práctica financiera.

La opinión de los contadores públicos sobre la aplicación móvil con base en el procesamiento de datos por medidas de tendencia central, los resultados muestran un nivel de calidad excelente debido a que se cumple con la variable de funcionalidad, porque proporcionar funciones apropiadas para realizar las tareas o actividades financieras; en cuanto a la eficiencia, el tiempo de respuesta, el recurso utilizado es el apropiado y cumple con los requisitos normativos para procesamiento de la información mediante la utilización en dispositivo móvil lo hace practico y disponible en cualquier momento; por ser compatible para el intercambio de información o llevar a cabo las funciones requeridas en el contexto financiero; su uso promueve el entendimiento, aprendizaje y resulta atractivo para el profesionista al momento de realizar la tareas financieras por medio de dispositivos electrónicos; es confiable por que las funciones especificadas bajo condiciones requeridas por las normatividad y el periodo de tiempo determinados satisfacer la necesidades propias de la actividad; por su seguridad, se protege la información susceptible de ser modificada o alterado por personal no autorizado; su mantenibilidad hace que sea una aplicación susceptible de ser modificada durante su uso de operación y por su portabilidad hace de la aplicación que pueda ser utilizada en cualquier momento y en cualquier lugar al momento que se requiere; así mismo los resultados muestran que existe una opinión unificada entre los participantes, es decir que todos los contadores opinión que el nivel de calidad es excelente de la aplicación para generar la información financiera sobre el manejo y control 
del efectivo.

En la correlación de Pearson al existir un alto grado de relación entre todas las variables independientes en relación con la variable dependiente; se encontró que al aumentar la funcionalidad, eficiencia, compatibilidad, usabilidad, confiabilidad, seguridad, mantenibilidad y portabilidad aumenta la calidad, es evidente que la aplicación móvil es práctico, eficiente relacionado, fácil de usar, confiable, seguro, factible y puede trasladarse a cualquier lugar y en cualquier momento para realizar las tareas o actividades relacionadas en el contexto financiero con calidad.

En la regresión lineal los resultados muestran que la influencia es significancia en cada una de las variables independiente con respecto a la variable dependiente; en encontró que las funciones contendías, el recurso como dispositivos tecnológico, la compatibilidad con las actividades, el uso del teléfono inteligentes, la confianzas de su contenido, la accesibilidad y la ejecución de la aplicación y su portabilidad, en su conjunto hacen la materialización de la calidad se muestre en aplicación puesta en la práctica financiera.

Los coeficientes de determinación $\left(\mathrm{r}^{2}\right)$ permitieron conocer las variables independientes funcionalidad, eficiencia y portabilidad presentan proporciona de variación significativa con respecto a la variable dependiente de calidad; es decir que las funciones contendidas en la aplicación, el tipo de dispositivo electrónico y la transportación son las variables son relevadores de la calidad de la aplicación financiera.

\section{CONCLUSIONES}

La aplicación móvil financiera posee un excelente nivel de calidad debido a que cumple con los criterios de la norma ISO 25010, el uso de esta tecnología permite el cumplimiento de las funciones requeridas para la realización de sus actividades, siendo el tiempo de respuesta inmediato, es practica y portable, cumple con los criterios que marca la normatividad lo que lo hace confiable, permitiendo al usuario su fácil comprensión, aprendizaje y entendimiento, por ello resulta atractivo para el profesionista en la realización de sus funciones financieras, brinda seguridad al usuario pues satisface las necesidades propias de la actividad al ser susceptible de modificaciones durante su uso de operaciones, 
lo cual permite al profesionista la realización pertinente, oportuna, relevante, confiable, eficiente y eficaz de su labor profesional.

Para el profesionista el uso de tecnología en su actividad profesional se facilita por la oportuna obtención de resultados confiables, puesto que se ahorra tiempo y esfuerzo, proporcionando servicios de alta calidad, promoviendo la competitividad, productividad y desarrollo organizacional lo cual impacta en sus ingresos económicos; además el contar con tecnología que contiene calidad internacional y normatividad vigente es un recurso de competitividad en el mercado que enmarca la diferencia en la prestación de servicio proporcionando información segura confiable y oportuna que hacen que el incremento de la eficiencia y eficacia de la utilización del recurso sea un detonante intrínseco en su labor profesional.

Los trabajos futuros sobre la misma línea de investigación consisten en generar aplicaciones tecnológicas que se apeguen a las normas internacionales de calidad así como a las disposiciones legales vigentes que promuevan las diferentes áreas que abarcan las normas de educación continua profesional con el propósito de construir un auto conocimiento sustentado en el juicio profesional con la visión de mejorar los procesos gubernamentales, empresariales y académicos que requiere la misma profesión.

\section{REFERENCIAS}

ABC Economía, (2013), Las aplicaciones en los móviles son ya un instrumento laboral más, consultado el 15 de diciembre 2019. https://www.abc.es/economia/20131120/abciaplicaciones-moviles-instrumento-laboral-201311191705.html

Benítez \& Ramírez (2013), "Evaluación de la calidad sobre la aplicación móvil fiscal para cálculo de la PTU en empresas mexicanas", RIDE, vol. 3, núm. 6, enero-junio, 2013, pp. 219-236 recuperado de https://www.redalyc.org/pdf/4981/498150314013.pdf

IABMx, (2012), "Estudio de usos y hábitos de dispositivos móviles", MillwardBrown, primera edición, agosto, consultado el 28 de noviembre del 2019. http://www.iabmexico.com/wpcontent/uploads/2016/02/IABMx.Estudio-UHMobile2012.pdf

International Standards Organization, (2000). NOM 9000. Organización Internacional De Normalización. https://www.ioe-emp.org/es/organizaciones-internacionales/organizacioninternacional-para-la-normalizacion/

International Standards Organization, (1994). NOM 8402. Organización Internacional De Normalización. https://www.ioe-emp.org/es/organizaciones-internacionales/organizacioninternacional-para-la-normalizacion/ 
Monsalve, L. (2004), "Calidad de los Productos Software". Consultado el 11 de noviembre del 2019. http://dialnet.unirioja.es/servlet/articulo?codigo=2098142

Moreno, S.; González, C. \& Echartea, C. (2008), "Evaluación de la Calidad en Uso de Sitios Web Asistida por Software: $S W-A Q U A$ ”. Avances en Sistemas e Informática, vol. 5, núm. 1, mayo, 2008, pp. 147-154. Universidad Nacional de Colombia.

Omaña, M. y Cadenas, J. (2010). Manufactura Esbelta: una contribución para el desarrollo de software con calidad. Enl@ce Revista Venezolana de Información, Tecnología y Conocimiento, 7 (3), 11-26.

Pressman, R. (2010). Ingeniería del Software. Un enfoque práctico. 7ta edición. España: Ed: McGraw-Hill Interamericana.

Piattini V. M. G. , Garcia, R. F.O. \& Caballero M-R. I. (2006). Calidad de Sistemas Informáticos. p. 416. Editorial Alfaomega

Real Academia Española, (2020), Diccionario de la lengua española, 23. a ed., [calidad]. Consultado el 8 de enero 2020. https://dle.rae.es .

Rodríguez, M.; Verdugo, J.; Coloma, R.; Genero, M. \& Piattini, M. (2010). Metodología para la evaluación de la calidad en los modelos UML. REICIS Revista Española de Innovación, Calidad e Ingeniería del Software, Vol. 6, Núm. 1, abril-sin mes, 2010, pp. 16-35. Asociación de Técnicos de Informática. España.

Santoveña, S. (2010). Cuestionario de evaluación de la calidad de los cursos virtuales de la UNED. RED. Revista de Educación a Distancia, núm. 25, 2010, pp. 1-22. Universidad de Murcia. Murcia, España.

Solarte, G.; Muñoz, L. \& Arias, B. (2009); Modelos de calidad para procesos de software. Scientia Et Technica, vol. XV, núm. 42, agosto, 2009, pp. 375-379. Universidad Tecnológica de Pereira. Colombia. https://www.redalyc.org/pdf/849/84916714069.pdf

Scalone, F. (2006). Estudio comparativo de los modelos y estándares de calidad del software. [Tesis Ingeniería de Calidad]. Repositorio institucional: Universidad Tecnológica Nacional Regional de Buenos Aires. 488 p. 